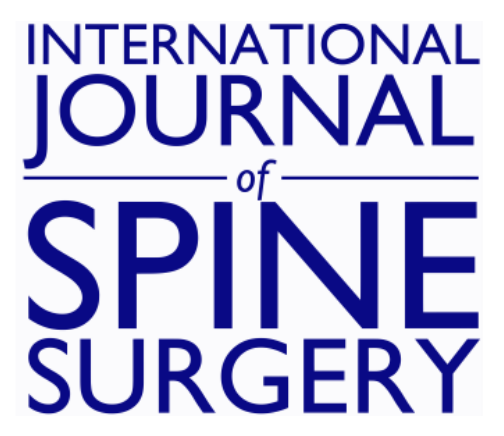

\title{
Spinal Anesthesia for Geriatric Lumbar Spine Surgery: A Comparative Case Series
}

NOAH L. LESSING, CHARLES C. EDWARDS II, CLAYTON L. DEAN, OLIVE H. WAXTER, CHARLES LIN, RYAN A. CURTO and CHARLES H. BROWN IV

Int J Spine Surg 2020, 14 (5) 713-721

doi: https://doi.org/10.14444/7103

http://ijssurgery.com/content/14/5/713

This information is current as of April 26, 2023.

Email Alerts Receive free email-alerts when new articles cite this article. Sign up at:

http://ijssurgery.com/alerts

The International Journal of Spine Surgery

2397 Waterbury Circle, Suite 1,

Aurora, IL 60504, Phone: +1-630-375-1432 


\title{
Spinal Anesthesia for Geriatric Lumbar Spine Surgery: A Comparative Case Series
}

\author{
NOAH L. LESSING, BS, ${ }^{1}$ CHARLES C. EDWARDS II, MD, ${ }^{1}$ CLAYTON L. DEAN, MD,${ }^{1}$ OLIVE H. WAXTER,

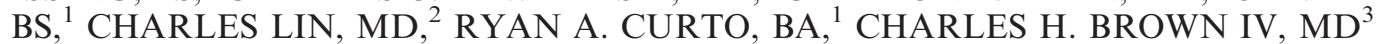 \\ ${ }^{I}$ The Maryland Spine Center and ${ }^{2}$ Department of Anesthesiology, Mercy Medical Center, Baltimore, Maryland, ${ }^{3}$ Department of Anesthesiology and Critical Care \\ Medicine, Johns Hopkins University School of Medicine, Baltimore, Maryland
}

\begin{abstract}
Background: The use of spinal anesthesia (SA) as opposed to general anesthesia (GA) during elective lumbar spine surgery is an emerging technique and represents a potentially modifiable factor to limit perioperative complications. Few studies, however, have compared these anesthetic techniques in an elderly population. The aim of this study is to determine if SA is a safe alternative to GA for lumbar spine surgery in elderly patients.

Methods: A retrospective, consecutive case series study was performed. All patients aged 70 years and older who underwent lumbar spine decompression or combined decompression and fusion using either SA or GA during a 2-year period at a single institution were identified. Demographics and perioperative outcomes were compared.

Results: Of all patients meeting the inclusion criteria, 56 patients $(19 \%)$ received SA and $239(81 \%)$ received GA. Patients receiving SA were slightly older (median age, 77 years versus 75 years, $P=.002)$, consisted of more men $(57 \%$ versus $36 \%, P=.01$ ), and had a lower mean body mass index (28.3 versus $30.1, P=.03)$. Indications for surgery and type of surgery were similar between groups. On average, operative times with SA were 101 minutes versus 103 minutes with GA $(P=.71)$. After controlling for age, sex, and body mass index, patients receiving SA had decreased estimated blood loss $(\beta=-75 \mathrm{~mL} ; 95 \%$ confidence interval $[\mathrm{CI}],-140.6,-9.4 ; P=.025)$ and intraoperative intravenous fluid requirements $(\beta=-205 \mathrm{~mL} ; 95 \% \mathrm{CI},-389.4,-21.0 ; P=.029)$, shorter postanesthesia care unit stays $(\beta=-41 \mathrm{minutes}$; $95 \% \mathrm{CI},-64.6,-16.9 ; P=.001)$, lower maximum visual analog scale pain scores $(\beta=-0.89$ points; $95 \% \mathrm{CI},-1.6,-0.1 ; P$ $=.020$ ), and decreased odds of receiving blood transfusion (odds ratio, $0.12 ; 95 \% \mathrm{CI}, 0.01,0.62 ; P=.45$ ); there were no significant differences in operative time, length of stay, nausea, or oral morphine equivalents consumed per day. Complication rates were similar between groups.

Conclusion: Spinal anesthesia is a reasonable, safe alternative to general anesthesia for lumbar spine surgery in elderly patients with degenerative conditions.

Lumbar Spine

Keywords: spinal anesthesia, geriatrics, lumbar fusion, lumbar decompression
\end{abstract}

\section{INTRODUCTION}

General anesthesia (GA) is the most commonly used technique to anesthetize patients during spine surgery. Regularly used during hip and knee procedures, the use of spinal anesthesia (SA) during elective lumbar spine surgery is an emerging technique. ${ }^{1}$ The choice between SA and GA represents a potentially modifiable factor to limit perioperative complications with lumbar spine surgery.

Several publications have suggested that SA is a safe alternative to GA for adults undergoing lumbar decompression procedures, ${ }^{2-7}$ and may even have perioperative benefits. ${ }^{8}$ Few studies, however, report on the application of SA for spine surgery in the geriatric population, ${ }^{9-12}$ despite the burgeoning number of elderly patients receiving spine surgery. ${ }^{13}$
Given that elderly patients are at increased anesthetic risk because of their lack of physiologic reserve, they are a particularly appealing population for less burdensome anesthesia regimens.

The purpose of this study is to determine whether $\mathrm{SA}$ is a reasonable, safe alternative to GA for spine surgery in an elderly population. Perioperative outcomes and complications of SA and GA in a consecutive series of elderly patients (aged 70 years or older) undergoing lumbar spine decompression or combined decompression and fusion procedures at a single institution were compared.

\section{PATIENTS AND METHODS}

With Institutional Review Board approval, we retrospectively identified a consecutive series of 
patients from a prospectively collected database who were aged 70 years or older and had undergone lumbar decompression or decompression/fusion by 1 of 2 board-certified orthopaedic surgeons with SA or GA at our department between December 2013 and October 2015. Patients with a diagnosis of spine tumor, infection, or fracture were excluded. Patients' operative reports, perioperative data, hospital discharge summaries, and other pertinent medical records were reviewed.

Patients given SA were determined preoperatively to be reasonable candidates by their surgeon and anesthesiologist. Generally, those at high risk for GA-related complications (eg, those experiencing cognitive decline or with major cardiovascular conditions) were offered both options. Patient preference from prior surgical experience and the authors' growing interest in SA also played a role in anesthetic selection. Contraindication for SA included severe aortic stenosis, prior pan-lumbar fusion, psychiatric disease that would preclude cooperation with sedation, or an airway at risk for obstruction with prone positioning. Every patient receiving SA was managed by a group of anesthesia providers at our institution who had a unique interest in and experience with SA.

SA was administered in the following manner: with the patient sitting, up to $100 \mu \mathrm{g}$ of fentanyl was used as premedication, and subcutaneous tissues were injected with lidocaine. The spinal needle was advanced towards the spinal canal at the L3 to L4 level. The selected level was shifted cephalad to L2 to L3 or caudal to L4 to L5 or L5 to S1 based on the surgical location and the levels previously fused. For decompression surgery, $50 \mathrm{mg}$ of $2 \%$ lidocaine was injected into the subarachnoid space. For combined decompression/fusion surgery, this was replaced with $15 \mathrm{mg}$ of $0.5 \%$ isobaric bupivacaine and 0.3 $\mathrm{mg}$ of preservative-free morphine sulfate. Needle placement was guided by anatomic landmarks, without supplemental radiographic guidance.

Once the SA was injected, the patient was positioned supine on the stretcher. When sensation was lost from the waist distally, the patient was positioned prone on a Jackson table (OSI, Union City, California) allowing for a free-hanging abdomen, or a Wilson frame (OSI) in an expanded position, according to surgeon preference, and given supplemental oxygen via nasal cannula. After confirmation of a stable airway, mild sedation was titrated with propofol based on the preference of the patient and comfort level of the anesthesiologist. Sedation to some extent was administered for all patients. If carbon dioxide monitoring alerted an obstruction in the patient's airway, the anesthesiologist inserted a nasopharyngeal airway. Patients were intermittently encouraged to move their neck and arms during the procedure to optimize comfort.

To maintain steady, spontaneous respiration, our SA paradigm stresses minimal use of intraoperative narcotics, and it avoids intraoperative benzodiazepine administration. This paradigm is designed to limit suppression of respiratory drive and decrease the possibility of apnea. Aspiration risk is reduced compared with spinal anesthesia in the supine position because gravity allows potential secretions or vomit to depart with gravity through the mouth while in the prone position.

Neuromonitoring was not used because SA interrupts voltage-gated sodium channels in both motor and sensory neural synapses, effectively reducing transmission through the neuroaxis.

The surgeon performed either a decompression or a combined decompression/fusion procedure via a posterior approach. Decompression consisted of a single-level or multilevel laminectomy, laminotomy, or partial discectomy, based on the stenotic pathology. All fusions received supplemental instrumentation using titanium rods and bilateral pedicle screws. A posterior intertransverse arthrodesis was performed for all patients undergoing combined decompression/fusion, with certain patients receiving interbody arthrodesis. Bone graft options included autogenous iliac crest, local bone, cancellous allograft, and/or recombinant human bone morphogenetic protein-2 (BMP; Infuse, Medtronic Sofamor Danek, Memphis, Tennessee). The choice of bone graft varied based on surgeon preference and patient-specific factors, such as nonunion risk, nutritional status and bone mineral density. Supplemental steroids or local anesthetics were not used at the time of closure for any patient.

Decompressions were either performed as inpatient or outpatient procedures. All patients undergoing fusions were admitted to the hospital. Visual analog scale (VAS) pain scores and nausea (yes or no) were routinely obtained by nursing staff every 15 to 30 minutes in the postanesthesia care unit (PACU), and every 8 hours thereafter until hospital discharge. Maximum VAS was the highest reported score, from 0 to 10 , during the patient's hospital 
stay. Patients were positive for postoperative nausea if they responded yes at least once.

Postoperative narcotic requirements were converted into oral morphine equivalents (OMEs) for comparison purposes using an online calculator with standardized conversion ratios, ${ }^{14}$ used by Armaghani et al. ${ }^{15}$ Requirements were stratified by OMEs administered in the PACU per hour, and OMEs administered as an inpatient per day.

Hospital records were reviewed to determine the presence of intraoperative complications and complications occurring prior to discharge. Clinic notes from postoperative follow-ups and any documented postoperative communications were examined to determine the occurrence of complications presenting after hospital discharge. These events were defined as either major or minor. Major complications included cardiorespiratory arrest, respiratory failure, pulmonary embolism (PE), acute myocardial infarction (MI), arrhythmia requiring intervention, stroke/transient ischemic attack (TIA), wound infection requiring reoperation, hematoma requiring reoperation, hospital readmission for failure to thrive, bacterial pneumonia, aspiration pneumonia, and pneumonia with unknown organism. Other adverse events were defined as minor complications.

Statistical testing was performed with RStudio, version 0.98.1091. Continuous, parametric variables were analyzed using Welch $t$ tests. Two-by-two contingency tables were analyzed using Fisher exact tests if any cell value was equal to or less than 5, and Pearson $\chi^{2}$ tests with Yates continuity correction if all cell values were greater than 5. Pearson $\chi^{2}$ tests were used for analyzing contingency tables with dimensions larger than two-by-two. Outcomes of SA versus GA were compared using multivariate regression controlling for age, sex, and body mass index (BMI). For all tests, $P$ values $\leq 0.05$ were considered significant.

Values are presented in tables as mean $\pm \mathrm{SD}$ (range), mean $\pm \mathrm{SD}$ (median, range), or frequency $(\%)$ unless otherwise noted.

\section{RESULTS}

\section{Demographics}

Between December 2013 and October 2015, a total of 295 consecutive patients 70 years or older underwent lumbar spine decompression or decompression/fusion by 1 of 2 surgeons at a single institution for degenerative pathology. Of these, 56 $(19 \%)$ received SA and $239(81 \%)$ received GA.
Patients receiving SA were slightly older (median age, 77 years versus 75 years, $P=.002)$, male predominant $(57 \%$ versus $36 \%, P=.01)$, and had a lower mean BMI (28.3 versus 30.1, $P=.03$; Table 1). Patients in both groups had a similar mean Charlson comorbidity index (CCI; SA, 4.20; GA, 4.44; $P=.19$ ) and ASA status (SA, 2.68; GA, 2.57; $P$ $=.27$ ). SA and GA groups were also similar in their indications for surgery, percentage undergoing combined decompression and fusion (SA, 52\%; GA, $65 \% ; P=.51$ ), and percentage undergoing revision surgery (SA, 25\%; GA, 37\%; $P=.12$ ).

\section{Surgical Characteristics}

Operations in the SA group spanned, on average, 1.82 vertebral levels (range, 1-4), compared with 1.94 levels (range, $1-5)$ in the GA group $(P=.35)$. The L4 to L5 level was most frequently operated on in both groups. The most common surgeries in both groups were combined decompression/fusions of either L4 to L5 or L4 to S1.

Intraoperative cerebrospinal fluid (CSF) leaks occurred at a similar rate in both groups (SA, 5\%; GA, $14 \% ; P=.11$; Table 1$)$. All cases were fully repaired at the time they were noted. In the SA group, all 3 spinal fluid leaks arose from dural laceration during decompression, not from puncture with the spinal needle. More procedures using SA were done as outpatient surgeries compared with GA $(27 \%$ versus $13 \%, P=.02)$.

\section{Outcomes}

Mean operating time was similar between SA (101 minutes) and GA (103 minutes) patients $(P=$ .71; Table 1). GA patients had greater estimated blood loss (EBL; 269 versus $187 \mathrm{~mL} ; P=.01$ ), and received more intraoperative intravenous (IV) fluids (976 versus $753 \mathrm{~mL}, P=.001$ ). No SA patients converted to GA.

SA patients spent less time in the PACU (mean, 123 versus 171 minutes, $P=.001$ ), reported lower maximum VAS scores from incision closure through hospital discharge (mean, 6.21 versus 7.39, $P=$ .002 ), and had a lower frequency of blood transfusion during their hospital stay $(2 \%$ versus $13 \%, P=$ .02 ; Table 1). Length of stay, occurrence of nausea, and amount of OMEs consumed per hour in the PACU and per day as an inpatient did not differ between groups. SA and GA patients experienced similar rates of complications. 
Table 1. Characteristics and outcomes of spinal anesthesia (SA) versus general anesthesia (GA) for lumbar spine surgery in elderly patients 70 years and older.

\begin{tabular}{|c|c|c|c|}
\hline Characteristic & $\mathrm{SA}(\mathrm{n}=56)$ & GA $(n=239)$ & $P$ Value ${ }^{a}$ \\
\hline \multicolumn{4}{|l|}{ Demographics } \\
\hline Age, mean $\pm \mathrm{SD}$ (range), $\mathrm{y}$ & $78.0 \pm 5.5(77,70-91)$ & $75.5 \pm 4.4(75,70-92)$ & $.00 * * *$ \\
\hline Male, No. (\%) & $32(57)$ & $87(36.4)$ & $.01 * * *$ \\
\hline $\mathrm{BMI}$, mean $\pm \mathrm{SD}$ (range) & $28.3 \pm 5.6(17.2-47.3)$ & $30.1 \pm 5.8(16.6-49.8)$ & $.03 * * *$ \\
\hline $\mathrm{CCI}$, mean $\pm \mathrm{SD}$ (median, range) & $4.20 \pm 1.20(4,3-8)$ & $4.44 \pm 1.30(4,3-10)$ & .19 \\
\hline $\mathrm{ASA}$ mean $\pm \mathrm{SD}$ (median, range) & $2.68 \pm 0.66(3,2-4)$ & $2.57 \pm 0.57(3,1-4)$ & .27 \\
\hline Taking antiplatelet or anticoagulant, No. (\%) & $28(50)$ & $133(55.6)$ & .54 \\
\hline Sleep apnea, No. $(\%)$ & $6(10.7)$ & $27(11.3)$ & 1.00 \\
\hline \multicolumn{4}{|l|}{ Primary diagnosis, No. $(\%)$} \\
\hline Stenosis & $23(41.1)$ & $64(26.8)$ & .05 \\
\hline Disk herniation & $4(7.1)$ & $19(7.9)$ & 1.00 \\
\hline Spondylolisthesis & $19(33.9)$ & $86(36.0)$ & .89 \\
\hline Severe foraminal stenosis & $3(5.4)$ & $29(12.1)$ & .23 \\
\hline Scoliosis & $4(7.1)$ & $13(5.4)$ & .54 \\
\hline Severe adjacent segment disease & $1(1.8)$ & $23(9.6)$ & .06 \\
\hline Recurrent disk herniation & $1(1.8)$ & $2(0.8)$ & .47 \\
\hline Nonunion & $1(1.8)$ & $3(1.3)$ & .57 \\
\hline Total & & & .12 \\
\hline \multicolumn{4}{|l|}{ Surgical characteristics } \\
\hline Combined decompression/fusion, No. (\%) & $29(51.8)$ & $156(65.3)$ & .51 \\
\hline Revision, No. (\%) & $14(25.0)$ & $89(37.2)$ & .12 \\
\hline Interbody, No. $(\%)$ & $22(39.3)$ & $117(49.0)$ & .25 \\
\hline Intraoperative CSF leak, No. (\%) & $3(5.4)$ & $33(13.8)$ & .11 \\
\hline Operative time, mean $\pm \mathrm{SD}$ (range), min & $101 \pm 42(30-210)$ & $103 \pm 48(30-300)$ & .71 \\
\hline $\mathrm{EBL}$, mean $\pm \mathrm{SD}$ (range), $\mathrm{mL}$ & $187 \pm 190(20-700)$ & $269 \pm 219(20-1500)$ & $.01 * * *$ \\
\hline Intraoperative IV fluids, mean $\pm \mathrm{SD}$ (range), $\mathrm{mL}$ & $753 \pm 404(73-1669)$ & $976 \pm 622(0.5-3112)$ & $.00 * * *$ \\
\hline Outpatient, No. $(\%)$ & $15(26.8)$ & $31(13.0)$ & $.02 * * *$ \\
\hline Time in PACU, mean $\pm \mathrm{SD}$ (range), min & $123 \pm 58(32-310)$ & $171 \pm 83(36-716)$ & $.00 * * *$ \\
\hline Length of stay, mean $\pm \mathrm{SD}$ (median, range), days & $2.41 \pm 1.30(3,1-6)$ & $2.69 \pm 1.48(3,1-11)$ & .16 \\
\hline Nausea, No. $(\%)$ & $12(21.4)$ & $48(20.1)$ & .97 \\
\hline Max VAS score, $0-10$, mean $\pm \mathrm{SD}$ (median, range) & $6.21 \pm 2.50(6,0-10)$ & $7.39 \pm 2.49(8,0-10)$ & $.00 * * *$ \\
\hline OMEs in PACU per hour, mean $\pm \mathrm{SD}$ (range) & $8.38 \pm 13.6(0-64.8)$ & $5.66 \pm 7.23(0-45.7)$ & .15 \\
\hline OMEs as inpatient per day, mean $\pm \mathrm{SD}$ (range) & $34.8 \pm 32.0(0-161.7)$ & $37.5 \pm 36.5(0-282.5)$ & .64 \\
\hline Blood transfusion, No. (\%) & $1(1.8)$ & $30(12.6)$ & $.02 * * *$ \\
\hline \multicolumn{4}{|l|}{ Complications, No. $(\%)^{\mathrm{b}}$} \\
\hline Total & $10(17.9)$ & $63(26.4)$ & .25 \\
\hline Major & $3(5.4)$ & $10(4.2)$ & .72 \\
\hline Minor & $7(12.5)$ & $53(22.2)$ & .15 \\
\hline Thromboembolic & $0(0)$ & $11(4.6)$ & .13 \\
\hline Urologic & $2(3.6)$ & $21(8.8)$ & .27 \\
\hline Cardiac & $3(5.4)$ & $5(2.1)$ & .18 \\
\hline Neurologic & $2(3.6)$ & $6(2.5)$ & .65 \\
\hline PE & $0(0)$ & $2(0.8)$ & 1.00 \\
\hline MI & $1(1.8)$ & $0(0)$ & .19 \\
\hline Arrhythmia requiring intervention & $1(1.8)$ & $0(0)$ & .19 \\
\hline Stroke/TIA & $0(0)$ & $2(0.8)$ & 1.00 \\
\hline Readmitted for failure to thrive & $0(0)$ & $2(0.8)$ & 1.00 \\
\hline Wound complication requiring surgical debridement & $1(1.8)$ & $3(1.3)$ & .57 \\
\hline Hematoma requiring surgery & $0(0)$ & $1(0.4)$ & 1.00 \\
\hline DVT & $0(0)$ & $9(3.8)$ & .22 \\
\hline UTI & $1(1.8)$ & $12(5.0)$ & .47 \\
\hline Urinary retention & $1(1.8)$ & $9(3.8)$ & .69 \\
\hline Arrhythmia & $1(1.8)$ & $5(2.1)$ & 1.00 \\
\hline Positional headaches & $0(0)$ & $1(0.4)$ & 1.00 \\
\hline CSF leak repair & $0(0)$ & $1(0.4)$ & 1.00 \\
\hline Altered mental status & $2(3.6)$ & $3(1.3)$ & .24 \\
\hline Syncopal episode & $0(0)$ & $2(0.8)$ & 1.00 \\
\hline Transient foot drop & $1(1.8)$ & $1(0.4)$ & .34 \\
\hline Clostridium difficile & $0(0)$ & $1(0.4)$ & 1.00 \\
\hline Peripheral edema & $0(0)$ & $1(0.4)$ & 1.00 \\
\hline Yeast infection & $0(0)$ & $4(1.8)$ & 1.00 \\
\hline Wound cellulitis & $1(1.8)$ & $4(1.8)$ & 1.00 \\
\hline
\end{tabular}

Abbreviations: ASA, American Society of Anesthesiologists; BMI, body mass index; CCI, Charlson comorbidity index; CSF, cerebrospinal fluid; DVT, deep vein thrombosis; EBL, estimated blood loss; IV, intravenous; MI, myocardial infarction; OME, oral morphine equivalent; PACU, postanesthesia care unit; PE, pulmonary embolism; TIA, transient ischemic attack; UTI, urinary tract infection; VAS, visual analog scale.

${ }^{a}$ Asterisks denote statistically significant at $P \leq .05$.

${ }^{\mathrm{b}}$ Major complications are bolded. 
Table 2. Comparing outcomes of spinal anesthesia (SA) versus general anesthesia (GA) for lumbar spine surgery in elderly patients 70 years and older; multivariate regression controlled for age, sex, and body mass index.

\begin{tabular}{|c|c|c|c|c|}
\hline \multirow[b]{2}{*}{ Variable } & \multirow[b]{2}{*}{ Regression Type } & \multicolumn{2}{|c|}{ Multivariate Regression } & \multirow{2}{*}{$\frac{\text { Favorable Technique }}{\text { SA/GA/Similar }}$} \\
\hline & & $\mathrm{OR} / \beta(95 \% \mathrm{CI})$ & $P$ Value $^{\mathrm{a}}$ & \\
\hline Operative time $(\mathrm{min})$ & Linear $(\beta)$ & $0.94(-13.17,15.06)$ & .896 & Similar \\
\hline Estimated blood loss (mL) & Linear $(\beta)$ & $-75.01(-140.61,-9.41)$ & $.025^{* * *}$ & SA \\
\hline Intraoperative IV fluids (mL) & Linear $(\beta)$ & $-205.23(-389.44,-21.03)$ & $.029 * * *$ & SA \\
\hline Time in PACU $(\mathrm{min})$ & Linear $(\beta)$ & $-40.71(-64.55,-16.86)$ & $.001 * * *$ & SA \\
\hline Length of stay (days) & Linear $(\beta)$ & $-0.14(-0.57,0.30)$ & .534 & Similar \\
\hline Nausea & Logistic (OR) & $1.18(0.54,2.46)$ & .664 & Similar \\
\hline Max VAS score $(0-10)$ & Linear $(\beta)$ & $-0.89(-1.63,-0.14)$ & $.020 * * *$ & SA \\
\hline OMEs in PACU per hour & Linear $(\beta)$ & $2.69(0.03,5.35)$ & $.048 * * *$ & GA \\
\hline OMEs as inpatient per day & Linear $(\beta)$ & $-0.29(-12.74,12.16)$ & .964 & Similar \\
\hline Blood transfusion & Logistic (OR) & $0.12(0.01,0.62)$ & $.045^{* * *}$ & SA \\
\hline
\end{tabular}

Abbreviations: CI, confidence interval; IV, intravenous. OME, oral morphine equivalents; OR, odds ratio; PACU, postanesthesia care unit; VAS, visual analog scale. ${ }^{a}$ Asterisks denote statistically significant at $P \leq 0.05$.

When controlling for age, sex, and BMI, SA patients had decreased EBL by $75 \mathrm{~mL}(95 \%$ confidence interval $[\mathrm{CI}],-140.6,-9.4 ; P=.025)$ and intraoperative IV fluid requirements by $205 \mathrm{~mL}$ (95\% CI, -389.4, $-21.0 ; P=.029$; Table 2). SA patients spent 41 minutes less in the PACU $(95 \%$ CI, $-64.6,-16.9 ; P=.001)$ and consumed 2.7 OMEs more per hour in the PACU $(95 \% \mathrm{CI}, 0.03,5.4 ; P=$ .048). Maximum VAS scores throughout hospital stay were 0.89 points lower among SA patients (95\% CI, $-1.6,-0.1 ; P=.020)$. Patients receiving SA had decreased odds of receiving a blood transfusion while in the hospital (odds ratio, 0.12; 95\% CI, 0.01, $0.62 ; P=.45)$.

\section{Subanalyses of Decompression Only and Combined Decompression/Fusion Procedures}

Subanalyses of patients undergoing decompression only (SAD versus GAD) and combined decompression/fusion (SADF versus GADF) were also performed. Compared with the group GAD, group SAD was slightly older (median, 78 versus 75 years, $P=.02$ ), had less EBL (67 versus $118 \mathrm{~mL}, P=$ $.01)$, and spent less time in the PACU (130 versus 165 minutes, $P=.03$; Table 3 ). All other measurables between groups were similar, including frequency of complications. Compared with group GADF, group SADF had more men $(55 \%$ versus $33 \%, P=.04)$, received less intraoperative IV fluids (930 versus $1215 \mathrm{~mL}, P=.004$ ), spent less time in the PACU (117 versus 174 minutes, $P=.001$ ), and had lower postoperative VAS pain scores (6.7 versus 8.1, $P=.001$; Table 4). The rest of the variables, including frequency of complications, were similar between groups.

\section{DISCUSSION}

We report a single institution's experience with patients 70 years and older undergoing lumbar spine surgery using either SA or GA. The results of this retrospective, consecutive case series show that SA is a reasonable, safe alternative to GA in elderly patients receiving lumbar spine decompression or decompression/fusion procedures. This study bolsters previous observations that SA is feasible for spine surgery in the geriatric population, and expands the literature by comparing its use in lengthy decompression/fusion procedures to a GA cohort.

Until recently, investigations of SA for spine surgery have focused on the adult population. A meta-analysis of randomized controlled trials comparing SA to GA for adults undergoing lumbar decompression suggested that SA may hold perioperative benefits over GA. ${ }^{8}$ The meta-analysis showed that patients receiving SA experience less postoperative nausea and vomiting, shorter lengths of stay, and less intraoperative EBL, despite similar surgical durations. The incidence of intraoperative hypotension and postoperative urinary retention was similar between anesthetic techniques.

The first study of SA for spine surgery in the geriatric population presented a case series of 56 patients 70 years and older (median, 77 years; range, 70-91 years) receiving lumbar spine decompression or combined decompression/fusion. ${ }^{12}$ The novel report was the first to show the safe use of SA for spine surgery in patients older than 84 years and for surgeries lasting up to 3.5 hours. All of the patients survived, and there were no instances of stroke, PE, permanent loss of function, or conversion to general anesthesia. No cases required supplemental intra- 
Table 3. Characteristics and outcomes of spinal anesthesia (SAD) versus general anesthesia (GAD) for lumbar spine decompression in elderly patients 70 years and older.

\begin{tabular}{|c|c|c|c|}
\hline Characteristic & SAD $(\mathbf{n}=27)$ & GAD $(\mathbf{n}=83)$ & $P$ Value $^{\mathrm{a}}$ \\
\hline \multicolumn{4}{|l|}{ Demographics } \\
\hline Age, mean $\pm \mathrm{SD}$ (median, range) & $79.4 \pm 6.2(78,70-91)$ & $76.2 \pm 5.1(75,70-92)$ & $.02 * * *$ \\
\hline Male, No. $(\%)$ & $16(59)$ & $36(43)$ & .22 \\
\hline BMI, mean \pm SD (range) & $28.2 \pm 6.0(17.2-47.3)$ & $29.9 \pm 5.2(16.6-41.2)$ & .20 \\
\hline $\mathrm{CCI}$, mean $\pm \mathrm{SD}$ (median, range) & $4.15 \pm 1.13(4,3-7)$ & $4.24 \pm 1.17(4,3-8)$ & .72 \\
\hline ASA, mean \pm SD (median, range) & $2.67 \pm 0.55(3,2-4)$ & $2.54 \pm 0.55(3,1-4)$ & .33 \\
\hline Taking antiplatelet or anticoagulant & $16(59)$ & $46(55)$ & .90 \\
\hline Sleep apnea, No. $(\%)$ & $2(7)$ & $5(6)$ & 1.00 \\
\hline \multicolumn{4}{|l|}{ Primary diagnosis, No. (\%) } \\
\hline Stenosis & $23(85)$ & $64(77)$ & .43 \\
\hline Disk herniation & $4(15)$ & $19(23)$ & .43 \\
\hline \multicolumn{4}{|l|}{ Surgical characteristics } \\
\hline Revision, No. (\%) & $3(11)$ & $18(22)$ & .27 \\
\hline Intraoperative CSF leak, No. (\%) & $1(4)$ & $9(11)$ & .45 \\
\hline Operative time, mean $\pm \mathrm{SD}$ (range), min & $70 \pm 24(30-120)$ & $60 \pm 22(30-170)$ & .06 \\
\hline $\mathrm{EBL}$, mean $\pm \mathrm{SD}$ (range), $\mathrm{mL}$ & $67 \pm 85(20-400)$ & $118 \pm 110(20-600)$ & $.01 * * *$ \\
\hline Intraoperative IV fluids, mean $\pm \mathrm{SD}$ (range), $\mathrm{mL}$ & $555 \pm 253(144-1271)$ & $530 \pm 323(0.5-1310)$ & .69 \\
\hline Outpatient, No. (\%) & $15(56)$ & $31(37)$ & .15 \\
\hline Time in PACU, mean \pm SD (range), mins & $130 \pm 65(44-310)$ & $165 \pm 83(43-401)$ & $.03 * * *$ \\
\hline Length of stay, mean $\pm \mathrm{SD}$ (median, range), days & $1.67 \pm 1.27(1,1-6)$ & $1.43 \pm 0.95(1,1-5)$ & .39 \\
\hline Nausea, No. (\%) & $3(11)$ & $14(17)$ & .66 \\
\hline Max VAS score, mean \pm SD (range), $0-10$ & $5.70 \pm 2.83(0-10)$ & $6.02 \pm 2.92(0-10)$ & .61 \\
\hline OMEs in PACU per hour, mean $\pm \mathrm{SD}$ (range) & $12.0 \pm 17.8(0-64.8)$ & $5.0 \pm 6.0(0-26.8)$ & .05 \\
\hline OMEs as inpatient per day, mean \pm SD (range) & $38.1 \pm 50.8(0-161)$ & $32.1 \pm 29.4(0-150)$ & .66 \\
\hline Blood transfusion, No. $(\%)$ & $0(0)$ & $0(0)$ & 1.00 \\
\hline \multicolumn{4}{|l|}{ Complications, No. $(\%)^{\mathrm{b}}$} \\
\hline Total & $5(18.5)$ & $14(16.9)$ & 1.00 \\
\hline Major & $1(3.7)$ & $3(3.6)$ & 1.00 \\
\hline Minor & $4(14.8)$ & $11(13.3)$ & 1.00 \\
\hline Thromboembolic & $0(0)$ & $4(4.8)$ & .57 \\
\hline Urologic & $1(3.7)$ & $5(6.0)$ & 1.00 \\
\hline Cardiac & $1(3.7)$ & $0(0)$ & .25 \\
\hline Neurologic & $1(3.7)$ & $0(0)$ & .25 \\
\hline PE & $0(0)$ & $1(1.2)$ & 1.00 \\
\hline Wound complication requiring surgical debridement & $1(3.7)$ & $1(1.2)$ & .43 \\
\hline Hematoma requiring surgery & $0(0)$ & $1(1.2)$ & 1.00 \\
\hline DVT & $0(0)$ & $3(3.6)$ & 1.00 \\
\hline UTI & $0(0)$ & $2(2.4)$ & 1.00 \\
\hline Urinary retention & $1(3.7)$ & $3(3.6)$ & 1.00 \\
\hline Arrhythmia & $1(3.7)$ & $0(0)$ & .25 \\
\hline CSF leak repair & $0(0)$ & $1(1.2)$ & 1.00 \\
\hline Altered mental status & $1(3.7)$ & $0(0)$ & .25 \\
\hline Transient foot drop & $1(3.7)$ & $1(1.2)$ & .43 \\
\hline Wound cellulitis & $0(0)$ & $1(1.2)$ & 1.00 \\
\hline
\end{tabular}

Abbreviations: ASA, American Society of Anesthesiologists; BMI, body mass index; CCI, Charlson comorbidity index; CSF, cerebrospinal fluid; DVT, deep vein thrombosis; EBL, estimated blood loss; IV, intravenous; MI, myocardial infarction; OME, oral morphine equivalent; PACU, postanesthesia care unit; PE, pulmonary embolism; TIA, transient ischemic attack; UTI, urinary tract infection; VAS, visual analog scale.

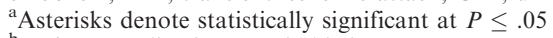

${ }^{\mathrm{b}}$ Major complications are bolded.

operative spinal anesthetic. Unfortunately, there was no GA cohort for comparison.

The second study of SA for spine surgery in the geriatric population compared outcomes of lumbar decompression in 146 SA patients to those in $292 \mathrm{GA}$ patients. ${ }^{16}$ The SA group was selected based on high cardiovascular and/or pulmonary risk (American Society of Anesthesiologists [ASA] score II-IV). There was no ASA cutoff for the GA group. All surgeries were less than 90 minutes, and patients with diagnoses of spondylolisthesis or scoliosis were excluded. The SA group was significantly older (75 years versus 69 years), had a lower mean BMI (25.8 versus 27.2) and had a higher mean ASA score (3 versus 2). Patients receiving SA experienced decreases in: (1) duration of surgery, (2) number of hypotensive events, (3) amount of intraoperative vasopressors, (4) EBL, (5) transfusion rate, (6) VAS in PACU, (7) time in PACU, (8) nausea and vomiting, and (9) total cost. Complication rates were similar between groups. The authors concluded that SA is superior to GA for high-risk patients undergoing spine surgery. Despite the positive findings of these 2 studies, many clinicians still question whether SA is a viable option for spine procedures in the geriatric population, particularly for surgeries lasting upwards of 3 hours. 
Table 4. Characteristics and outcomes of spinal anesthesia (SADF) versus general anesthesia (GADF) for lumbar spine combined decompression and fusion in elderly patients 70 years and older.

\begin{tabular}{|c|c|c|c|}
\hline Characteristic & SADF $(n=29)$ & GADF $(n=156)$ & $P$ Value $^{\mathrm{a}}$ \\
\hline \multicolumn{4}{|l|}{ Demographics } \\
\hline Age, mean \pm SD (median, range) & $76.8 \pm 4.6(75,70-87)$ & $75.2 \pm 4.0(75,70-88)$ & 0.09 \\
\hline Male, No. (\%) & $16(55)$ & $51(33)$ & $0.04 * * *$ \\
\hline BMI, mean \pm SD (range) & $28.3 \pm 5.3(19.1-38.1)$ & $30.2 \pm 6.1(18.8-49.8)$ & 0.10 \\
\hline $\mathrm{CCI}$, mean $\pm \mathrm{SD}$ (median, range) & $4.21 \pm 1.33(4,3-8)$ & $4.54 \pm 1.35(4,3-10)$ & 0.27 \\
\hline ASA, mean \pm SD (median, range) & $2.69 \pm 0.76(3,2-4)$ & $2.59 \pm 0.58(3,1-4)$ & 0.49 \\
\hline Taking antiplatelet or anticoagulant, No. (\%) & $12(41)$ & $30(20)$ & 0.22 \\
\hline Sleep apnea, No. $(\%)$ & $4(14)$ & $22(14)$ & 1.00 \\
\hline \multicolumn{4}{|l|}{ Indication for fusion, No. (\%) } \\
\hline Spondylolisthesis & $19(66)$ & $86(55)$ & 0.40 \\
\hline Severe foraminal stenosis & $3(10)$ & $29(19)$ & 0.42 \\
\hline Scoliosis & $4(14)$ & $13(8)$ & 0.31 \\
\hline Severe adjacent segment disease & $1(3)$ & $23(15)$ & 0.13 \\
\hline Recurrent disk herniation & $1(3)$ & $2(1)$ & 0.40 \\
\hline Nonunion & $1(3)$ & $3(2)$ & 0.50 \\
\hline Total & & & 0.35 \\
\hline \multicolumn{4}{|l|}{ Surgical characteristics } \\
\hline Revision, No. (\%) & $11(38)$ & $71(46)$ & 0.58 \\
\hline Interbody, No. $(\%)$ & $22(76)$ & $117(75)$ & 1.00 \\
\hline Intraoperative CSF leak, No. (\%) & $2(7)$ & $24(15)$ & 0.38 \\
\hline Operative time, mean $\pm \mathrm{SD}$ (range), min & $130 \pm 32(80-210)$ & $127 \pm 41(50-300)$ & 0.61 \\
\hline $\mathrm{EBL}$, mean $\pm \mathrm{SD}$ (range), $\mathrm{mL}$ & $303 \pm 193(20-700)$ & $351 \pm 221(60-1500)$ & 0.24 \\
\hline Intraoperative IV fluids, mean $\pm \mathrm{SD}$ (range), $\mathrm{mL}$ & $930 \pm 434(73-1669)$ & $1215 \pm 612(5.9-3112)$ & $0.00 * * *$ \\
\hline Time in PACU, mean $\pm \mathrm{SD}$ (range), min & $117 \pm 51(32-240)$ & $174 \pm 83(36-716)$ & $0.00 * * *$ \\
\hline Length of stay, mean $\pm \mathrm{SD}$ (median, range), days & $3.10 \pm 0.90(3,2-6)$ & $3.40 \pm 1.25(3,2-11)$ & 0.20 \\
\hline Nausea, No. (\%) & $9(33)$ & $34(22)$ & 0.40 \\
\hline Max VAS score, mean \pm SD (median, range), 0-10 & $6.69 \pm 2.09(7,0-10)$ & $8.12 \pm 1.86(8,2-10)$ & $0.00 * * *$ \\
\hline OMEs in PACU per hour, mean \pm SD (range) & $5.0 \pm 6.8(0-22.6)$ & $6.0 \pm 7.8(0-45.9)$ & 0.48 \\
\hline OMEs as inpatient per day, mean $\pm \mathrm{SD}$ (range) & $33.1 \pm 30.0(5-74.2)$ & $39.3 \pm 38.4(0-282.5)$ & 0.22 \\
\hline Blood transfusion, No. $(\%)$ & $1(3)$ & $30(20)$ & 0.05 \\
\hline \multicolumn{4}{|l|}{ Complications, No. $(\%)^{\mathrm{b}}$} \\
\hline Total & $5(17.2)$ & $49(31)$ & 0.18 \\
\hline Major & $2(6.9)$ & $7(4.5)$ & 0.63 \\
\hline Minor & $3(10.3)$ & $42(27.0)$ & 0.06 \\
\hline Thromboembolic & $0(0)$ & $7(4.5)$ & 0.60 \\
\hline Urologic & $1(3.4)$ & $16(10.2)$ & 0.48 \\
\hline Cardiac & $2(6.9)$ & $5(3.2)$ & 0.30 \\
\hline Neurologic & $1(3.4)$ & $6(3.8)$ & 1.00 \\
\hline DVT & $0(0)$ & $6(3.8)$ & 0.59 \\
\hline PE & $0(0)$ & $1(0.6)$ & 1.00 \\
\hline UTI & $1(3.4)$ & $10(6.4)$ & 1.00 \\
\hline Urinary retention & $0(0)$ & $6(3.8)$ & 0.59 \\
\hline MI & $1(3.4)$ & $0(0)$ & 0.16 \\
\hline Arrhythmia requiring intervention & $1(3.4)$ & $0(0)$ & 0.16 \\
\hline Arrhythmia & $0(0)$ & $5(3.2)$ & 1.00 \\
\hline Stroke/TIA & $0(0)$ & $2(1.3)$ & 1.00 \\
\hline Positional headaches & $0(0)$ & $1(0.6)$ & 1.00 \\
\hline CSF leak repair & $0(0)$ & $0(0)$ & - \\
\hline Altered mental status & $1(3.4)$ & $3(1.9)$ & 0.50 \\
\hline Syncopal episode & $0(0)$ & $2(1.3)$ & 1.00 \\
\hline Readmitted for failure to thrive & $0(0)$ & $2(1.3)$ & 1.00 \\
\hline Clostridium difficile & $0(0)$ & $1(0.6)$ & 1.00 \\
\hline Peripheral edema & $0(0)$ & $1(0.6)$ & 1.00 \\
\hline Yeast infection & $0(0)$ & $4(2.6)$ & 1.00 \\
\hline Wound complication requiring surgical debridement & $0(0)$ & $2(1.3)$ & 1.00 \\
\hline Wound cellulitis & $1(3.4)$ & $3(1.9)$ & 0.50 \\
\hline
\end{tabular}

Abbreviations: ASA, American Society of Anesthesiologists; BMI, body mass index; CCI, Charlson comorbidity index; CSF, cerebrospinal fluid; DVT, deep vein thrombosis; EBL, estimated blood loss; IV, intravenous; MI, myocardial infarction; OME, oral morphine equivalent; PACU, postanesthesia care unit; PE, pulmonary embolism; TIA, transient ischemic attack; UTI, urinary tract infection; VAS, visual analog scale.

${ }^{a}$ Asterisks denote statistically significant at $P \leq 0.05$

${ }^{\mathrm{b}}$ Major complications are bolded.

In the current study, several lengthy procedures were reported: the SA group had a mean operative time of 101 minutes, with 21 cases lasting between 2 and 3 hours, and 5 lasting more than 3 hours. The safety and efficacy of SA observed with operative times of up to 3 hours and 30 minutes suggest that SA may be appropriate for longer periods than previously thought.

The current study demonstrated several superior outcomes for patients receiving SA. First, patients 
receiving SA were more hemodynamically stable during surgery. After controlling for age, sex, and BMI, SA patients had decreased intraoperative fluid requirements, EBL, and transfusion rate. Theoretically, SA blocks sympathetic signaling causing vasodilation, hypotension, and subsequent retention of intravascular and extravascular fluid, which is one potential explanation for these findings. Multivariate regression also revealed that SA patients reported lower maximum VAS scores during their hospital stay. Although lower pain scores in SA patients may have been driven by increased OMEs per hour in the PACU, the OMEs consumed per day across the inpatient stay were similar. Lastly, SA patients spent less time in the PACU. Improved postoperative efficiency and transfer to the spine surgery inpatient unit among SA patients could be a function of improved intraoperative hemodynamics and subjective pain severity.

Importantly, none of the measured outcomes favored GA over SA, aside from SA patients consuming more OMEs per hour in the PACU per hour. There was no difference in the frequency of major, minor, thromboembolic, urologic, cardiac, neurologic, or specific complications between groups. In GA patients, there was a $4 \%$ occurrence of major complications (PE, stroke/TIA, hospital readmission for failure to thrive, wound infection requiring reoperation, and hematoma requiring reoperation) versus $5 \%$ in SA patients (acute MI, arrhythmia requiring intervention, and wound infection requiring reoperation). The frequency of minor complications was also similar regardless of anesthesia type.

SA may be an appealing option for those at high risk for postoperative delirium, which is associated with increased length of stay, hospital cost, and cognitive decline. ${ }^{17,18}$ Using a validated assessment method, 1 study reported its incidence to be $40.5 \%$ after spine surgery in older adults. ${ }^{19}$ Given that reduced depth of anesthesia decreases the incidence of postoperative delirium, ${ }^{20}$ spinal anesthesia could potentially lessen postoperative neurologic dysfunction. A limitation of the current study is that a formal delirium assessment was not carried out uniformly using a validated instrument.

Disadvantages of SA include the potential for CSF leak while injecting the anesthetic, and the short half-lives of the injectable agents. Symptomatic CSF leaks after spinal anesthesia are reported to occur at rates between $2 \%$ and $12 \%$ depending upon the size of the spinal needle. ${ }^{21}$ Fortunately, the rate of CSF leaks in the SA group was only $5 \%$, and none arose from spinal needle puncture. Because the half-lives of lidocaine and bupivicaine are 1.5 to 2 hours and 2.7 hours, respectively, SA could wear off prior to the end of the procedure. In this scenario, additional SA can be administered by the surgeon via an intrathecal injection within the surgical field. To date, such has not been necessary in our institution's experience of more than 200 patients with surgical durations up to 3 hours and 30 minutes. $^{22}$

The present study has several weaknesses. Given the study design, there may have been selection bias as reflected in demographic differences between groups, namely age, sex, and BMI. These differences were controlled for with multivariate regression when comparing outcomes. Additionally, long-term outcomes were not obtained but are provided elsewhere. ${ }^{23,24,25}$ Finally, despite the use of a prospective registry, the data were analyzed retrospectively.

Herein constitutes an accurate snapshot of one institution's experience using spinal anesthesia for elderly spine surgery-all consecutive patients meeting the selection criteria since the adoption of this technique by the surgeons were included in the analysis. Hospital records and clinic notes were available for $100 \%$ of patients in both groups, which is a particular strength of the study.

\section{CONCLUSION}

$\mathrm{SA}$ is a reasonable, safe alternative to GA for elderly patients undergoing spine surgery. Prospective randomized trials are necessary to further characterize the differences between both techniques in this population, and ultimately determine which technique is superior.

\section{REFERENCES}

1. De Rojas JO, Syre P, Welch WC. Regional anesthesia versus general anesthesia for surgery on the lumbar spine: a review of the modern literature. Clin Neurol Neurosurg. 2014;119:39-43.

2. Attari MA, Mirhosseini SA, Honarmand A, Safavi MR. Spinal anesthesia versus general anesthesia for elective lumbar spine surgery: a randomized clinical trial. J Res Med Sci. 2011;16(4):524-529.

3. Jellish JP, Thalji Z, Stevenson K, Shea J. A prospective randomized study comparing short- and intermediate-term perioperative outcome variables after spinal or general anes- 
thesia for lumbar disk and laminectomy surgery. Anesth Analg. 1996;83(3):559-564.

4. McLain RF, Kalfas I, Bell GR, et al. Comparison of spinal and general anesthesia in lumbar laminectomy surgery: a case-controlled analysis of 400 patients. J Neurosurg Spine. 2005;2(1):17-22.

5. Tetzlaff JE, O'Hara JF Jr, Yoon HJ, Schubert A. Spinal anesthesia for elective lumbar spine surgery. $J$ Clin Anesth. 1998;10(8):656-659.

6. Chen HT, Tsai CH, Chao SC, et al. Endoscopic discectomy of L5-S1 disc herniation via an interlaminar approach: prospective controlled study under local and general anesthesia. Surg Neurol Int. 2011;2:93.

7. McLain RF, Tetzlaff JE, Bell GR, et al. Microdiscectomy: spinal anesthesia offers optimal results in general patient population. J Surg Orthop Adv. 2007;16(1):5-11.

8. Zorrilla-Vaca A, Healy RJ, Mirski MA. A comparison of regional versus general anesthesia for lumbar spine surgery: a meta-analysis of randomized studies. J Neurosurg Anesthesiol. 2017;29(4):415-425.

9. Goddard M, Smith PD. Spinal anaesthesia for spinal surgery. Anaesthesia. 2006;61(7):723-724.

10. Singeisen H, Hodel D, Schindler C, et al. Significantly shorter anesthesia time for surgery of the lumbar spine: process analytical comparison of spinal anesthesia and intubation narcosis. Anaesthetist. 2013;62(8):632-638.

11. Erbas YC, Pusat S, Yilmaz E, et al. Posterior lumbar stabilization surgery under spinal anesthesia for high-risk patients with degenerative spondylolisthesis, spinal stenosis and lumbar compression fracture. Turk Neurosurg. 2015;25(5):771-775.

12. Lessing NL, Edwards CC 2nd, Brown $\mathrm{CH} 4$ th, et al. Spinal anesthesia in elderly patients undergoing lumbar spine surgery. Orthopedics. 2017;40(2):e317-e322.

13. Deiner S, Westlake B, Dutton RP. Patterns of surgical care and complications in elderly adults. $J$ Am Geriatr Soc. 2014;62(5):829-835.

14. McAuley D. Advanced opiod converter. 2013. Available at https://globalrph.com/medcalcs/advanced-opioid-conver sions-equianalgesic-morphine-equivalents/. Accessed February $1,2017$.

15. Armaghani SJ, Lee DS, Bible JE, et al. Preoperative opioid use and its association with perioperative opioid demand and postoperative opioid independence in patients undergoing spine surgery. Spine. 2014;39(25):e1524-1530.

16. Finsterwald M, Muster M, Farshad M, et al. Spinal versus general anesthesia for lumbar spine surgery in high risk patients: perioperative hemodynamic stability, complications, and costs. J Clin Anesth. 2018;46:3-7.

17. Saczynski JS, Marcantonio ER, Quach L, et al.
Cognitive trajectories after postoperative delirium. $N$ Engl $J$ Med. 2012; 367(1):30-39.

18. Rudolph JL, Marcantonio ER. Postoperative delirium: acute changes with long-term implications. Anesth Analg. 2011;112(5):1202-1211.

19. Brown CH 4th, LaFlam A, Max L, et al. Delirium after spine surgery in older adults: Incidence, risk factors, and outcomes. J Am Geriatr Soc. 2016;64(10):2101-2108.

20. Sieber FE. Postoperative delirium in the elderly surgical patient. Anesthesiol Clin. 2009;27(3):451-464.

21. Turnbull DK, Shepherd DB. Post-dural puncture headache: pathogenesis, prevention and treatment. $\mathrm{Br} J$ Anaesth. 2003;91(5):718-729.

22. Lessing NL, Edwards CC 2nd, Lin C, Brown CH 4th. Complex lumbar spine fusion for an elderly patient under spinal anesthesia. Orthopedics. 2017;40(5):e915-e917.

23. Deyo RA, Mirza SK, Martin BI, et al. Trends, major medical complications, and charges associated with surgery for lumbar spinal stenosis in older adults. JAMA. 2010;303(13):1259-1265.

24. Benz RJ, Ibrahim ZG, Afshar P, Garfin SR. Predicting complications in elderly patients undergoing lumbar decompression. Clin Orthop Relat Res. 2001;384:116-121.

25. Okuda S, Oda T, Miyauchi A, et al. Surgical outcomes of posterior lumbar interbody fusion in elderly patients. $J$ Bone Joint Surg Am. 2006;88(12):2714-2720.

Disclosures and COI: The authors do not have any proprietary interests in the materials described in this article, and do not report any conflicts of interest. The authors have not received grant support or research funding, except for Dr Charles H. Brown, in the form of Career Development Core of the Johns Hopkins Pepper Older Americans Independence Center, NIA P30AG021334.

Corresponding Author: Noah L. Lessing, BS, The Maryland Spine Center, Mercy Medical Center, 301 St Paul Pl, Baltimore, MD 21202. Phone: (631) 525-6395; Email: nlessing40@gmail.com.

Published 11 November 2020

This manuscript is generously published free of charge by ISASS, the International Society for the Advancement of Spine Surgery. Copyright (c) 2020 ISASS. To see more or order reprints or permissions, see http://ijssurgery.com. 